\title{
Study of Couple Stresses on MHD Poiseuille Flow through Porous Medium in a parallel plate channel
}

\author{
Raj Shekhar Prasad ${ }^{1} \&$ B. G. Prasad ${ }^{2}$ \\ ${ }^{l}$ Department of Mathematics, R. P. Sharma, Institute of Technology, Patna-801503, Bihar, India. \\ ${ }^{2}$ Department of Mathematics, B.N. College, Patna University, Patna-800004, Bihar, India.
}

\begin{abstract}
In this paper a mathematical analysis of effect of couple stresses on magneto hydrodynamic (MHD) flow past a parallel plate channel of viscous, incompressible and electrically conducting fluid in the presence of porous medium has been studied. An exact solution of the dimensionless governing equations has been obtained by using method of perturbation technique. The effects on velocity, discharge between the plates and wall shear are studied for different parameters like Hartmann number, Reynolds number, pulsation, time, porosity parameter, pulsation frequency and reported graphically to show the interesting aspect of the solution.
\end{abstract}

Keywords: Couple stresses, Magneto hydrodynamics (MHD), poiseuille flow, porous medium

\section{INTRODUCTION}

The theory of couple stress Fluid is developed by Stokes [22], defines the rotational field in terms of the velocity field for setting up the constitutive relationship between the stress and strain rate. The microcontinuum theory of Stokes is the simplest generalization of the classical theory of Fluids, which allows for polar effects such as the presence of couple stresses, body couples and a non-symmetric stress tensor. Some theoretical studies [1,16,17and 18] of blood flow indicate that some of the non-Newtonian flow properties of blood may be explained by assuming the blood to be a Fluid with couple stress. The couple stress Fluid may be considered as a special case of a non-Newtonian fluid which is intended to take into account the particle size effects. Moreover, the couple stress Fluid model is one of the numerous models that proposed to describe response characteristics of non-Newtonian Fluids. The constitutive equations in these Fluid models can be very complex and involving a number of parameters, also the out coming flow equations lead to boundary value problems in which the order of differential equations is higher than the Navier-Stokes equations. Some of the recent investigations regarding such Fluids are mentioned in the studies $[5,6,7,8,9,11,12$ and 13]. Chaturani has analyzed the problems of pulsatile flow of couple stress Fluid with application to blood flow [4]. An analysis is of the effects of couple stresses on the blood flow through thin artery with mild steno sis has been carried out by Sinha and Singh [20], Srivastava [21] considered the flow of couple stress Fluid through steno tic blood vessel.

Several authors [2, 10,14, 15 and 19] have discussed various aspects of unsteady hydrodynamic and hydro magnetic flows of an oldroyd-B Fluid both in a non-rotating rotating frame of reference..

With the above discussion in mind, the goal of this investigation is to study the effects of couple stress on unsteady hydro magnetic poiseuille flow in a saturated porous parallel-plate channel. Expressions for the velocity, non-dimensional discharge between the plate and Skin friction at wall have been obtained through the method of perturbation technique. The results have been discussed with the help of graphs.

\section{Mathematical Statement Of The Problem}

We consider the unsteady hydro magnetic flow of a viscous, incompressible and electrically conducting fluid induced by the pulsation of the pressure gradient. The plates are assumed to be electrically insulated. We also consider a Cartesian co-ordinate system $O X^{\prime} Y^{\prime} Z^{\prime}$ in such a way that the $X^{\prime} Z^{\prime}$-plane is taken on the lower plate and this $Y^{\prime}$-axis is normal to the plates. A uniform magnetic field $B_{O}$ is acting along the $Y^{\prime}$-axis. The induced magnetic field is assumed to be negligible. Within the framework of these assumptions, the equations which govern the MHD flow in the absence of both body force $f$ and body moment $I$ are

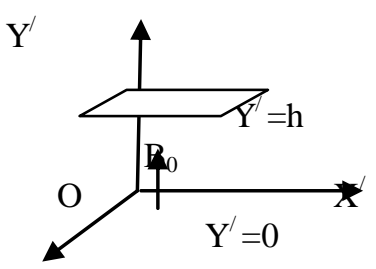




$$
\frac{\partial u^{\prime}}{\partial t^{\prime}}=-\frac{1}{\rho} \frac{\partial P^{\prime}}{\partial x^{\prime}}+\frac{\mu}{\rho} \frac{\partial^{2} u^{\prime}}{\partial y^{\prime 2}}-\frac{\eta}{\rho} \frac{\partial^{4} u^{\prime}}{\partial y^{\prime 4}}-\frac{\sigma B_{0}{ }^{2} u^{\prime}}{\rho}-\frac{\mu}{\rho k^{\prime}} u^{\prime}
$$

Here the term $-\frac{\eta}{\rho} \frac{\partial^{4} u^{\prime}}{\partial y^{\prime 4}}$ in this equation gives the effect of couple stresses, while $\frac{-\sigma B_{0}{ }^{2} u^{\prime}}{\rho}$ signifies the electromagnetic force and the term $\frac{-\mu}{\rho k^{\prime}} u^{\prime}$ due to porous media. All the physical quantities in the above equations have their usual meaning.

The boundary conditions corresponding to the problem under consideration are

$$
\begin{gathered}
u^{\prime}=0 \text { At } y^{\prime}=0 \\
u^{\prime}(1)=0 \text { At } y^{\prime}=h
\end{gathered}
$$

Since the couple stresses vanish at both the plates which in turn, implies that

$$
\begin{gathered}
\frac{\partial^{2} u^{\prime}}{\partial y^{\prime 2}}=0 \text { At } y^{\prime}=0 \\
\frac{\partial^{2} u^{\prime}}{\partial y^{\prime 2}}=0 \text { At } y^{\prime}=h
\end{gathered}
$$

Where $u^{\prime}(y, t)$ the fluid velocity field and $h$ is the distance between the two plates.

\subsection{Non-Dimensionlization}

We introduce the following dimensionless quantities

$$
\begin{aligned}
& u^{\prime}=v_{0} u, t^{\prime}=\frac{t h}{v_{0}}, P^{\prime}=\rho V_{0}^{2} P, x^{\prime}=x h ; y^{\prime}=y h, a^{2}=h^{2} / L^{2} L=\frac{h^{2}}{L^{2}}, \\
& L^{2}=\frac{\eta}{\mu}, R_{e}=\frac{V_{0} \rho h}{\mu}, \eta=\frac{\rho h^{4}}{V_{0}}, M^{2}=\frac{B_{0}{ }^{2} \sigma h^{2}}{\mu}, \omega^{\prime}=\frac{V_{0} \omega}{h}, \frac{1}{K}=\frac{h^{2}}{k^{\prime}}
\end{aligned}
$$

By using (6) in (1) - (5), we get

$$
\begin{gathered}
R_{e} a^{2} \frac{\partial u}{\partial t}=-R_{e} a^{2} \frac{\partial P}{\partial x}+a^{2} \frac{\partial^{2} u}{\partial y^{2}}-\frac{\partial^{4} u}{\partial y^{4}}-a^{2}\left(M^{2}+\frac{1}{K}\right) u \\
u=0, u^{\prime \prime}=0 a t y=0 ; u=0, u^{\prime \prime}=0 a t y=1
\end{gathered}
$$

Where prime denotes the differentiation with respect to $\mathbf{y}$.

\section{Mathematical Solution Of The Problem}

For solving equation (7), by using boundary conditions (8), we use the perturbation technique

$$
u=u_{s}+u_{o} e^{i \omega t}
$$

For pulsation pressure gradient, let

Using (10) in (7), we get

$$
-\frac{\partial P}{\partial x}=\left(\frac{\partial P}{\partial x}\right)_{s}+\left(\frac{\partial P}{\partial x}\right)_{o} e^{i \omega t}
$$

$$
R_{e} a^{2} \frac{\partial u}{\partial t}=R_{e} a^{2}\left[\left(\frac{\partial P}{\partial x}\right)_{S}+\left(\frac{\partial P}{\partial x}\right)_{o} e^{i \omega t}\right]+a^{2} \frac{\partial^{2} u}{\partial y^{2}}-\frac{\partial^{4} u}{\partial y^{4}}-a^{2}\left(M^{2}+\frac{1}{K}\right) u
$$

Substituting (9) in (7) and (8) and equating the like terms on both sides, we get the following system of equations:

$$
\frac{d^{4} u_{s}}{d y^{4}}-a^{2} \frac{d^{2} u_{s}}{d y^{2}}+a^{2}\left(M^{2}+\frac{1}{K}\right) u_{s}=R_{e} a^{2} P_{S}
$$




$$
\frac{d^{4} u_{o}}{d y^{4}}-a^{2} \frac{d^{2} u_{o}}{d y^{2}}+a^{2}\left(M^{2}+\frac{1}{K}+R_{e} i \omega\right) u_{o}=R_{e} a^{2} P_{O}
$$

Subject to the boundary conditions

$$
u_{s}=u_{o}=0, u_{s}^{\prime \prime}=u_{o}^{\prime \prime}=0 \text { aty }=0 ; \text { and } y=1
$$

The solutions of equations (12) and (13) subject to the boundary conditions (14) give the velocity distribution of the fluid under consideration:

$$
\begin{aligned}
u=( & \left.-N_{9} e^{\lambda_{11} y}+N_{8} e^{\lambda_{2} y}+N_{7} e^{\lambda_{3} y}+N_{6} e^{\lambda_{4} y}+\frac{R_{e} P_{S}}{\left(M^{2}+\frac{1}{K}\right)}\right) \\
& +\left[-N_{19} e^{\lambda_{5} y}+N_{18} e^{\lambda_{6} y}+N_{17} e^{\lambda_{7} y}+N_{16} e^{\lambda_{8} y}+\frac{R_{e} P_{O}}{\left(M^{2}+\frac{1}{K}+R_{e} i \omega\right)}\right] e^{i \omega t}
\end{aligned}
$$

Where

$$
\begin{aligned}
& \lambda_{1}=\left[\frac{1}{2}\left(a^{2}+\left(a^{4}-4 a^{2}\left(M^{2}+\frac{1}{K}\right)\right)^{\frac{1}{2}}\right)\right]^{\frac{1}{2}}, \lambda_{2}=-\lambda_{1} \\
& \lambda_{3}=\left[\frac{1}{2}\left(a^{2}-\left(a^{4}-4 a^{2}\left(M^{2}+\frac{1}{K}\right)\right)^{\frac{1}{2}}\right)\right]^{\frac{1}{2}}, \lambda_{4}=-\lambda_{3} \\
& \lambda_{5}=\left[\frac{1}{2}\left(a^{2}+\left(a^{4}-4 a^{2}\left(M^{2}+\frac{1}{K}+R_{e} i \omega\right)\right)^{\frac{1}{2}}\right)\right]^{\frac{1}{2}}, \lambda_{6}=-\lambda_{5} \\
& \lambda_{5}=\left[\frac{1}{2}\left(a^{2}-\left(a^{4}-4 a^{2}\left(M^{2}+\frac{1}{K}+R_{e} i \omega\right)\right)^{\frac{1}{2}}\right)\right]^{\frac{1}{2}}, \lambda_{8}=-\lambda_{7} \\
& \left(\frac{\partial P}{\partial x}\right)_{s}=P_{S},\left(\frac{\partial P}{\partial x}\right)_{o}=P_{O} \\
& N_{0}=\left(e^{\lambda_{1}}-e^{\lambda_{3}}\right)\left(\lambda_{2}^{2}-\lambda_{3}^{2}\right) \\
& N_{1}=\left(e^{\lambda_{1}}-e^{\lambda_{4}}\right)\left(\lambda_{2}^{2}-\lambda_{4}^{2}\right) \\
& N_{2}=\frac{R_{e} P_{S}}{\left(M^{2}+\frac{1}{K}\right)}\left(e^{\lambda_{1}}-1\right) \lambda_{2}^{2} \\
& N_{3}=\left(\lambda_{1}^{2} e^{\lambda_{2}}-\lambda_{2}^{2} e^{\lambda_{1}}\right)\left(\lambda_{1}^{2} e^{\lambda_{1}}-\lambda_{3}^{2} e^{\lambda_{3}}\right)-\left(\lambda_{1}^{2} e^{\lambda_{3}}-\lambda_{3}^{2} e^{\lambda_{1}}\right)\left(\lambda_{1}^{2} e^{\lambda_{1}}-\lambda_{2}^{2} e^{\lambda_{2}}\right) \\
& N_{4}=\left(\lambda_{1}^{2} e^{\lambda_{1}}-\lambda_{4}^{2} e^{\lambda_{4}}\right)\left(\lambda_{1}^{2} e^{\lambda_{2}}-\lambda_{2}^{2} e^{\lambda_{1}}\right)-\left(\lambda_{1}^{2} e^{\lambda_{4}}-\lambda_{4}^{2} e^{\lambda_{1}}\right)\left(\lambda_{1}^{2} e^{\lambda_{1}}-\lambda_{2}^{2} e^{\lambda_{2}}\right) \\
& N_{5}=\frac{R_{e} \lambda_{1}^{2} P_{S}}{\left(M^{2}+\frac{1}{K}\right)}\left[e^{\lambda_{1}}\left(\lambda_{1}^{2} e^{\lambda_{2}}-\lambda_{2}^{2} e^{\lambda_{1}}\right)-\left(\lambda_{1}^{2} e^{\lambda_{1}}-\lambda_{2}^{2} e^{\lambda_{2}}\right)\right] \\
& N_{6}=\frac{N_{5} N_{0}-N_{2} N_{3}}{N_{1} N_{3}-N_{4} N_{0}}, N_{7}=\frac{N_{2} N_{4}-N_{1} N_{5}}{N_{1} N_{3}-N_{0} N_{4}} \\
& N_{8}=\frac{1}{e^{\lambda_{2}}-e^{\lambda_{1}}}\left(\left(N_{7}\left(e^{\lambda_{1}}-e^{\lambda_{3}}\right)+N_{6}\left(e^{\lambda_{1}}-e^{\lambda_{4}}\right)+\frac{R_{e} P_{s}}{\left(M^{2}+\frac{1}{K}\right)}\left(e^{\lambda_{1}}-1\right)\right)\right. \\
& N_{9}=N_{6}+N_{7}+N_{8}+\frac{R_{e} P_{s}}{\left(M^{2}+\frac{1}{K}\right)}
\end{aligned}
$$




$$
\begin{aligned}
& N_{10}=\left(e^{\lambda_{5}}-e^{\lambda_{7}}\right)\left(\lambda_{6}^{2}-\lambda_{7}^{2}\right) \\
& N_{11}=\left(e^{\lambda_{5}}-e^{\lambda_{8}}\right)\left(\lambda_{6}^{2}-\lambda_{8}^{2}\right) \quad N_{12}=\frac{R_{e} P_{O}}{\left(M^{2}+\frac{1}{K}+R_{e} i \omega\right)}\left(e^{\lambda_{5}}-1\right) \lambda_{6}^{2} \\
& N_{13}=\left(\lambda_{5}^{2} e^{\lambda_{6}}-\lambda_{6}^{2} e^{\lambda_{5}}\right)\left(\lambda_{5}^{2} e^{\lambda_{5}}-\lambda_{7}^{2} e^{\lambda_{7}}\right)-\left(\lambda_{5}^{2} e^{\lambda_{7}}-\lambda_{7}^{2} e^{\lambda_{5}}\right)\left(\lambda_{5}^{2} e^{\lambda_{5}}-\lambda_{6}^{2} e^{\lambda_{6}}\right) \\
& N_{14}=\left(\lambda_{5}^{2} e^{\lambda_{5}}-\lambda_{8}^{2} e^{\lambda_{8}}\right)\left(\lambda_{5}^{2} e^{\lambda_{6}}-\lambda_{6}^{2} e^{\lambda_{5}}\right)-\left(\lambda_{5}^{2} e^{\lambda_{8}}-\lambda_{8}^{2} e^{\lambda_{5}}\right)\left(\lambda_{5}^{2} e^{\lambda_{5}}-\lambda_{6}^{2} e^{\lambda_{6}}\right) \\
& N_{15}=\frac{R_{e} \lambda_{5}^{2} P_{o}}{\left(M^{2}+\frac{1}{K}+R_{e} i \omega\right)}\left[e^{\lambda_{5}}\left(\lambda_{5}^{2} e^{\lambda_{6}}-\lambda_{6}^{2} e^{\lambda_{5}}\right)-\left(\lambda_{5}^{2} e^{\lambda_{5}}-\lambda_{6}^{2} e^{\lambda_{6}}\right)\right] \\
& N_{16}=\frac{N_{15} N_{10}-N_{12} N_{13}}{N_{11} N_{13}-N_{14} N_{10}}, N_{17}=\frac{N_{12} N_{14}-N_{11} N_{15}}{N_{11} N_{13}-N_{10} N_{14}} \\
& N_{18}=\frac{1}{e^{\lambda_{6}}-e^{\lambda_{5}}}\left(\left(N_{17}\left(e^{\lambda_{5}}-e^{\lambda_{7}}\right)+N_{16}\left(e^{\lambda_{5}}-e^{\lambda_{8}}\right)+\frac{R_{e} P_{o}}{\left(M^{2}+\frac{1}{K}+R_{e} i \omega\right)}\left(e^{\lambda_{5}}-1\right)\right)\right. \\
& N_{19}=N_{16}+N_{17}+N_{18}+\frac{R_{e} P_{o}}{\left(M^{2}+\frac{1}{K}+R_{e} i \omega\right)}
\end{aligned}
$$

The non-dimensional discharge between the plates per unit depth is given by $\mathrm{Q}$, where

$$
\begin{aligned}
Q= & \int_{0}^{1} u(y, t) d y \\
= & {\left[-\frac{N_{9}}{\lambda_{1}}\left(e^{\lambda_{1}}-1\right)+\frac{N_{8}}{\lambda_{2}}\left(e^{\lambda_{2}}-1\right)+\frac{N_{7}}{\lambda_{3}}\left(e^{\lambda_{3}}-1\right)+\frac{N_{6}}{\lambda_{4}}\left(e^{\lambda_{4}}-1\right)+\frac{R_{e} P_{S}}{\left.\left(M^{2}+\frac{1}{K}\right)\right]}\right.} \\
& +\left[-\frac{N_{19}}{\lambda_{5}}\left(e^{\lambda_{5}}-1\right)+\frac{N_{18}}{\lambda_{6}}\left(e^{\lambda_{6}}-1\right)+\frac{N_{17}}{\lambda_{7}}\left(e^{\lambda_{7}}-1\right)\right. \\
& \left.+\frac{N_{16}}{\lambda_{8}}\left(e^{\lambda_{8}}-1\right)+\frac{R_{e} P_{O}}{\left(M^{2}+\frac{1}{K}+R_{e} i \omega\right)}\right]
\end{aligned}
$$

Also the wall shear given in dimensionless form as

$$
\begin{aligned}
\tau_{w}=\left(\frac{d u}{d y}\right)_{y=1}= & {\left[-N_{9} \lambda_{1} e^{\lambda_{1}}+N_{8} \lambda_{2} e^{\lambda_{2}}+N_{7} \lambda_{3} e^{\lambda_{3}}+N_{6} \lambda_{4} e^{\lambda_{4}}\right]+} \\
& {\left[-N_{19} \lambda_{5} e^{\lambda_{5}}+N_{18} \lambda_{6} e^{\lambda_{6}}+N_{17} \lambda_{7} e^{\lambda_{7}}+N_{16} \lambda_{8} e^{\lambda_{8}}\right] e^{i \omega t} }
\end{aligned}
$$

Moreover when porosity parameter $K \rightarrow \infty$ the results agree with the corresponding ones of Nabil and Salwa [4]. 


\section{Results And Discussion}

The graphs (using MATLAB 7.0 software package) have been drawn to the inferences the velocity profiles of the pulsatile hydro magnetic poiseuille flow in a saturated porous medium with the effect of couple stresses, for several set of values of dimensionless parameters $K, P_{0}, P_{s}, a, t, R_{e} a$ and $\omega$. In Figs. 1-6, the influence of various parameters on velocity profile is shown. It can be observed from Fig. 1 that the velocity increases with increase of $P_{0}$ values. In fig. 2 it can be seen that the velocity decreases with increase in $\mathrm{K}$ value. It can be also seen that for fixed $\mathrm{K}$ the velocity increase in y initially and attains a maximum value closer to the upper plate. After achieving the maximum value, the velocity decreases with the increment in y. In fig. 3 it can be seen that the velocity decreases with increases in $\mathrm{K}$ value. It can be also seen that for fixed $\mathrm{K}$ the velocity increases in y initially and attains maximum velocity then decreases with the increment in $\mathrm{y}$ and then also increases with the increment in y. Fig.4 depict the influence of magnetic parameter M, on the velocity profile. As $\mathrm{M}$ increases from 10 to 40 , a considerable reduction in unsteady state velocity occurs.

From fig. 5 explicitly we can say that the velocity is maximum when $a$ is greater. In fig 6 we can say that the amplitude of velocity profile is maximum when $\omega=3$ minimum when $\omega=10$.

Figs. 7-8 depict the mass flux flow against $\mathrm{M}$ for various values of the pertinent parameters. Fig. 7 reveals the effect an increase in porosity $\mathrm{K}$ on the mass flow. In fig 8 it can be seen that when $R_{e}=20$ then mass flow increases with increase in $\mathrm{K}$ value. Figs. 9 and 10 concern with the wall Skin-friction against $\mathrm{M}$ for various values of pertinent parameters. In fig 9 we see that the partly portion of plots are linear and coincides with each other and then decreases with $\mathrm{K}$ increases. In Fig. 10 we observe that for $\mathrm{K}$ the skin-friction plot increases with increment in $\mathrm{y}$.

\section{References}

[1]. N. Ali, T. Hayat and M. Sajid, Peristaltic flow of a Couple stress fluid in an axisymmetric channel, Biorheology, 44, 2007, 125-138.

[2]. S. Asghar, S. Parveen, S. Hanif, A. M. Siddiqui and T. Hayat, Hall effects on the unsteady hydro magnetic flows of an Oldroyd-B fluid, International Journal of Engineering Science, 41, 2003, 609-619.

[3]. T. M. Nabil, EL-Dabe, Salba, and M.G. Mohandis, Effect of couple stresses on pulsatile hydromagnetic Poiseuille flow, Fluid Dynamics Research, 15(5), 1965, 313-324.

[4]. P. Chaturani and Upadhya, Pulsatile flow of a couple stress fluid through circular tubes with applications to blood flow, Biorheology, 15 (3-4) 1978, 193-201.

[5]. E. F. Elsehawey, A. M. Sobh, and N. A. S. Afifi, Peristaltic motion of a generalized Newtonian fluid under the effect of transverse magnetic field, Proc. of the Bulgarian Academy of Sciences 53, 2000, 33-38.

[6]. C. Fetecau, and C. Fetecau, on some axial Couette flows of non-. Newtonian fluids, Z. Math. Angew. Phys, 56, 2005, 1098-1106.

[7]. Abd El, Hakeem, Abd El, Naby and A. E. M. El. Misiery, Effects of an endoscope and generalized Newtonian fluid on peristaltic motion, Appl. Math. Com. 128, 2002, 19-35.

[8]. T. Hayat, N. Ali and S. Asghar, Peristaltic motion of a Burger's fluid in a planar channel, Appl. Math. Comput, 186 (1), 2007, 309329.

[9]. T. Hayat and N. Ali, Peristaltically induced motion of a MHD third grade fluid in a deformable tube, Physica A, 370(2), 2006, 225-239.

[10]. T. Hayat, S. Nadeem and S. Asghar, Hydromagnetic Couette flow of an Oldroyd-B fluid in a rotating system, International Journal of Engineering Science, 42, 2004, 65-78.

[11]. T. Hayat, Y. Wang, A. M. Siddiqui, K. Hutter and S. Asghar, Peristaltic transport of a third order fluid in a circular cylindrical tube, Math. Models Appl. Sci. 12, 2002, 1691-1706.

[12]. T. Hayat, A. H. Kara and E. Momoniat, Exact flow of a third grade-fluid on a porous wall, Int. J. Non-Linear Mech, 38, 2003, $1533-1537$.

[13]. T. Hayat, and A. H. Kara, Couette flow of a third-grade fluid with variable magnetic field, Mathematical Comput. Model, 43 2006, 132-137.

[14]. T. Hayat, A. M. Siddiqui and S. Asghar, Some simple flows of an Oldroyd-B fluid, International Journal of Engineering Science, 39, 2001, 135-147

[15]. C. T. Hsu, Dynamic modeling of convective heat transfer in porous media, in K. Vafai (Ed.), Hand book of porous media (second ed. Taylor and Francis, Boca Raton, FL, 2005), 39-80.

[16]. Kh. S. Mekheimer, Peristaltic flow of blood under effect of a magnetic field in a non uniform channels, Appl. Math. Comput.153, 2004, 763-777.

[17]. Kh. S. Mekheimer, Peristaltic transport of a couple stress fluids in uniform and non-uniform channels, Biorheology 39, 2002, 755765.

[18]. A. S. Popel, S. A. Regirer, and P. I. Usick, A continuum model of blood flow, Biorheology 11, 1974, $427-437$.

[19]. R. N. Ray, A. Samad, and T. K. Chaudhury, Low Reynolds number stability of MHD plane Poiseuille flow of an Oldroyd-B fluid, International Journal of Mathematics and Mathematical Sciences, 23(9), 2000, 617-625.

[20]. P. Sinha and C. Singh, Effects of couple stresses on blood flow through an artery with mild steno sis, Biorheology, 21(3), 1984 303315 .

[21]. L. M. Srivastava, Flow of couple stress fluid through steno tic blood vessels, J. Biomech, 18 (7), 1985, 479-485.

[22]. V. K. Stokes, Couple stresses in fluids, Phys. Fluids, 9, 1966, 1709-1715. 


\section{FIGURES:}

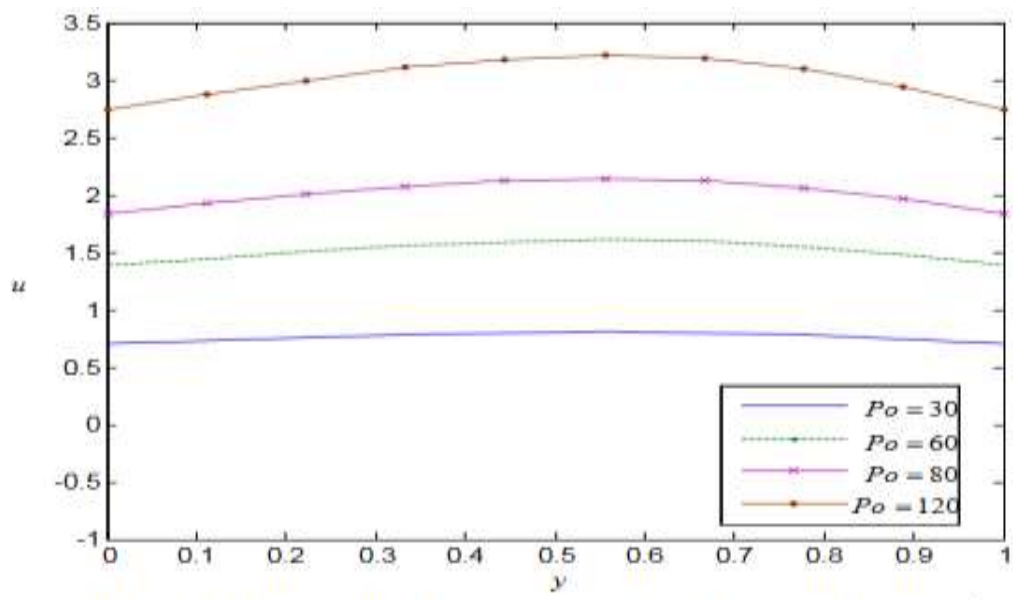

Figure 1 velocity distribution plotted versus position for $M-5, a-0.5, K-0.1, \omega-1, P_{s}-5, t-\pi / 2, \operatorname{Re}-2$.

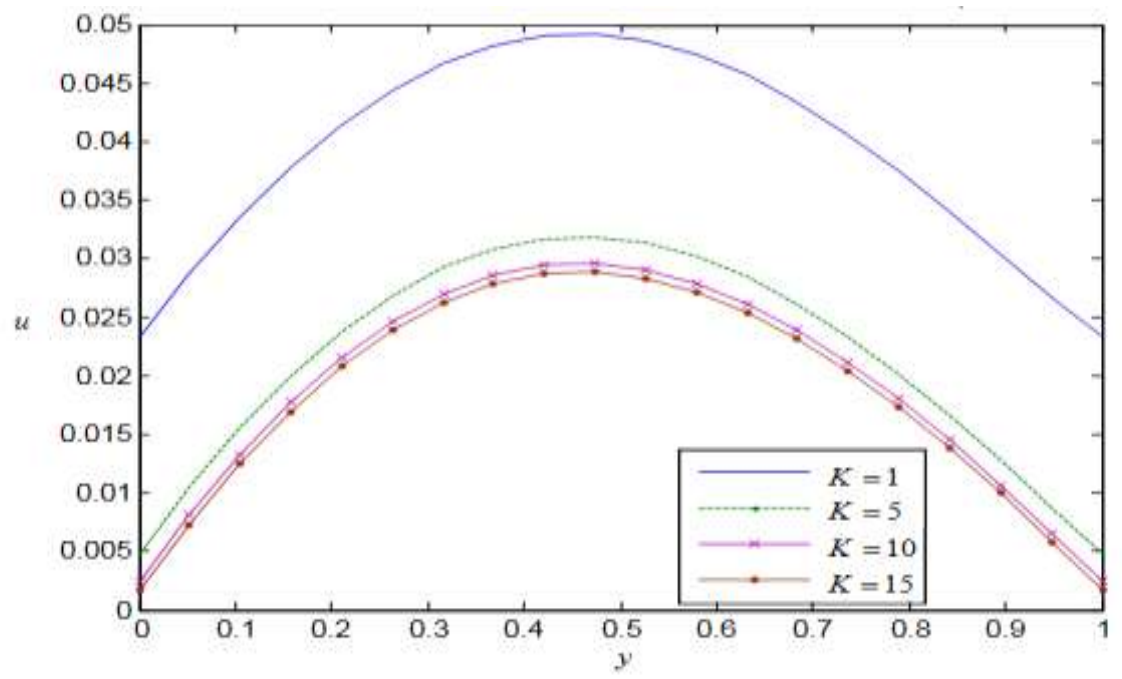

Figure 2 Velocity distribution plotted versus position for $M=5, a=0.5, P O=5, \omega=1, P S=5, t=\pi / 2, \operatorname{Re}=2$.

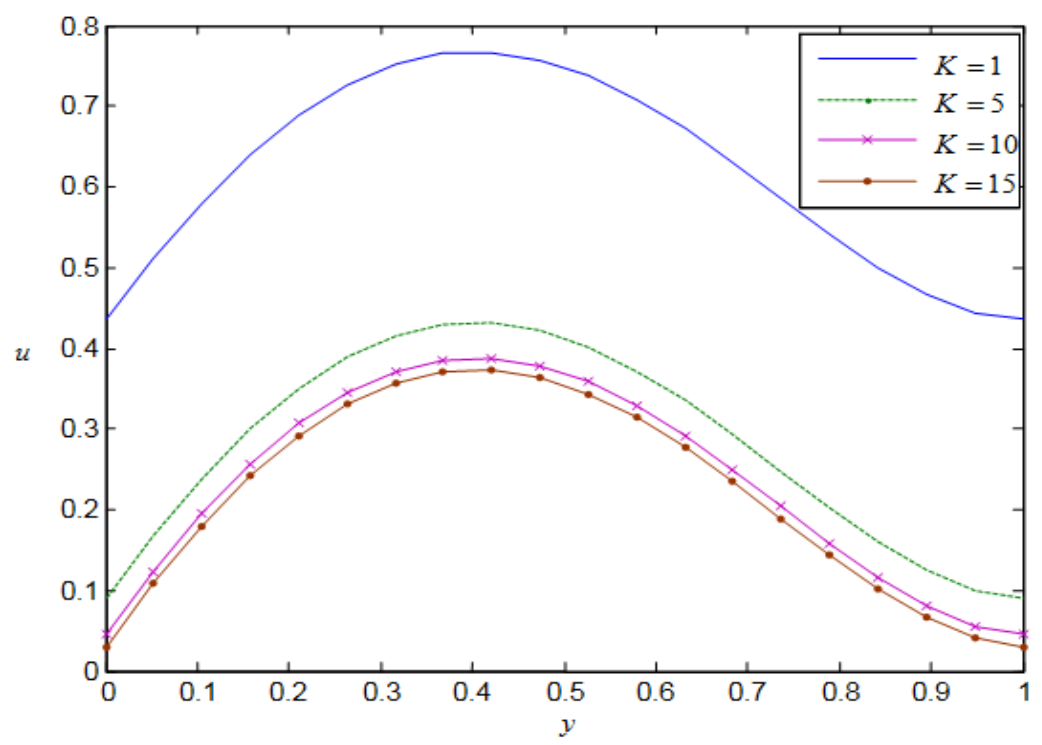

Figure 3 Velocity distribution plotted versus position for $M=5, a=0.5, P o=5, \omega=1, P s=5, t=\pi, \operatorname{Re}=50$. 


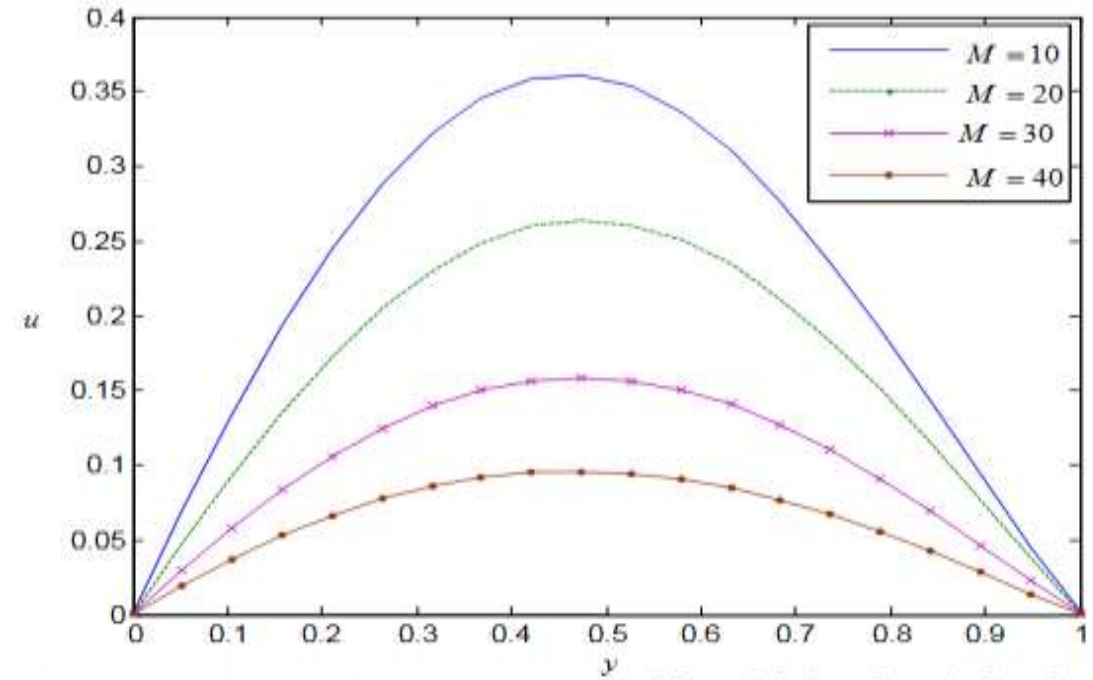

Figure 4 Velocity distribution plotted versus position for $K=10, a=0.5, P o=5, \omega=1, P s=5, t=\pi, \operatorname{Re}=50$.

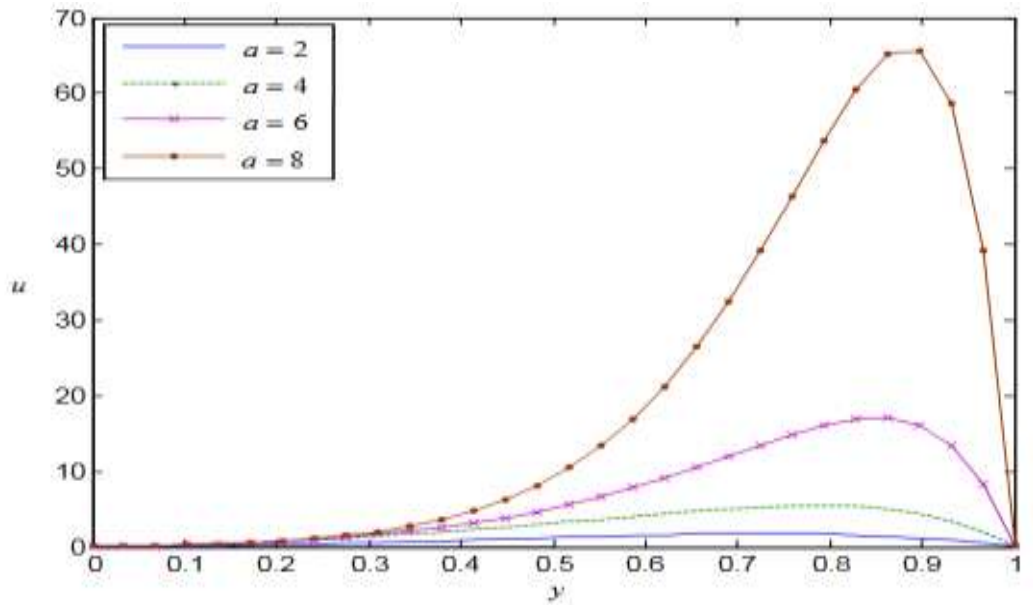

Figure 5 velocity dintribution plotted versun pasition for $K=10, M=4 Q, P O=5, \omega-1, P s-5, t-\pi$, Re -50

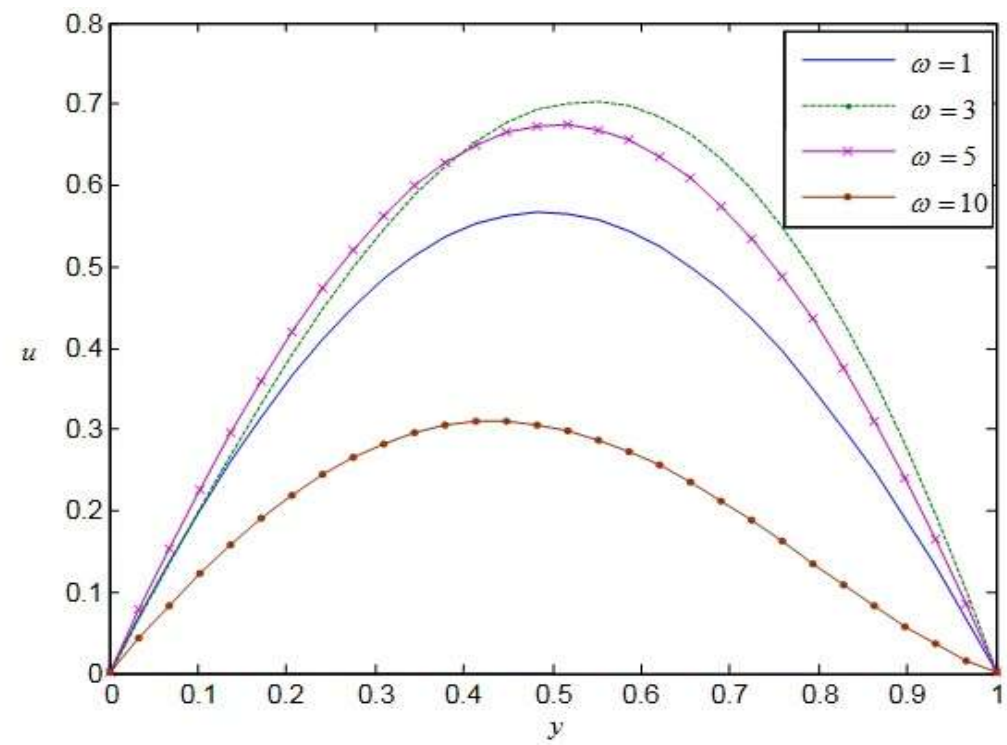

Figure 6 Velocity distribution plotted versus position for $K=1, M=10, P_{0}=5, a=1, P_{s}=5, t=\pi / 2, \operatorname{Re}=20$. 


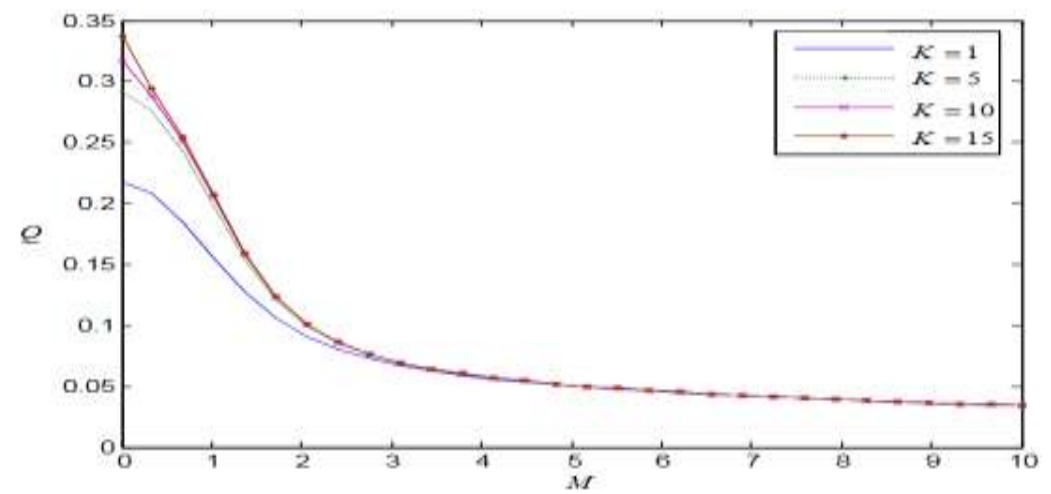

Finure 7 Divchurige $Q$ plotted vernun $M$ for $P O-2 Q a-0 . .5, P S-5, t=0.5$, Re $-2, c-1$.
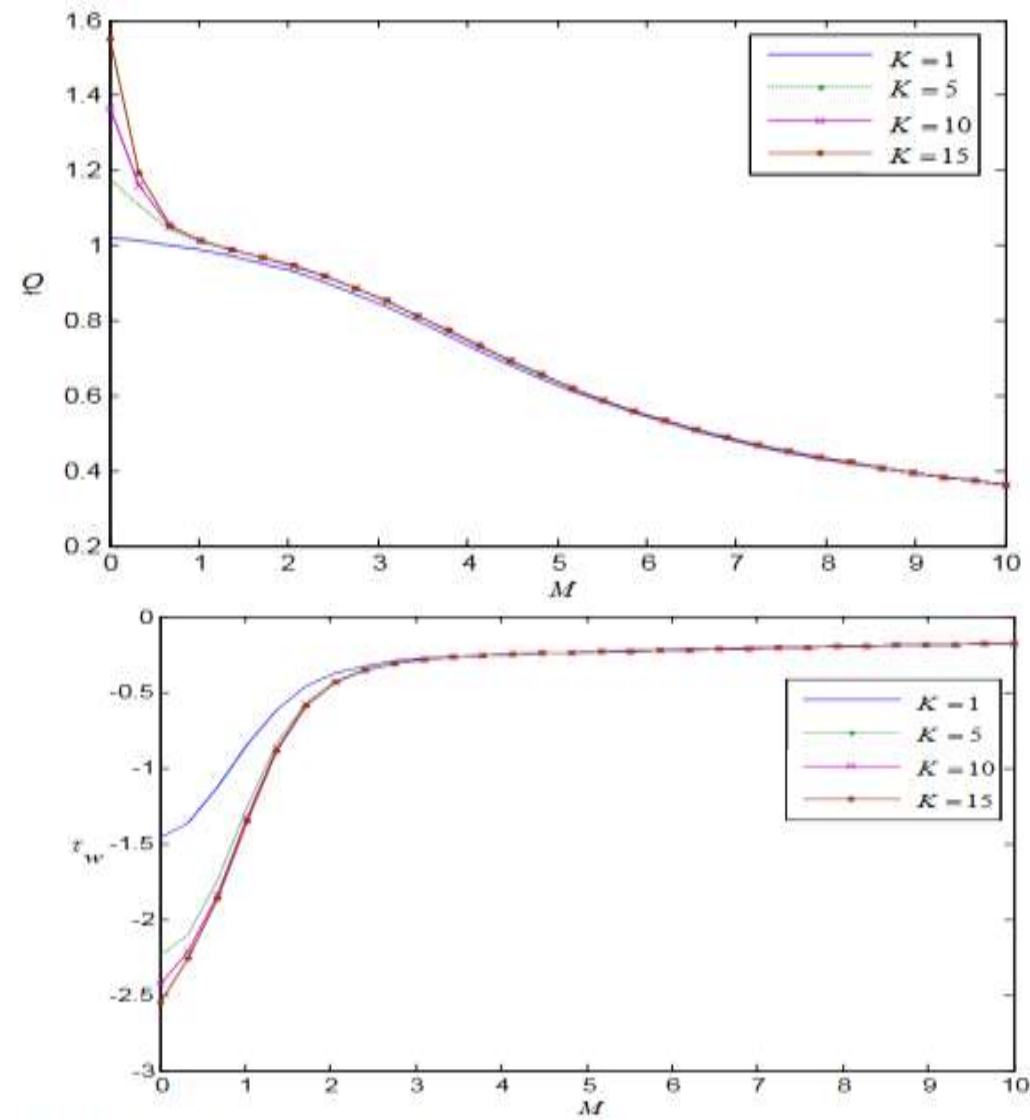

Figure 9 skin-friction $r_{1,}$ plotted versus $\mathrm{M}$ for $P O=20, a=0.5, P S=S, t=0.5$, Re $=2, \omega=1$.

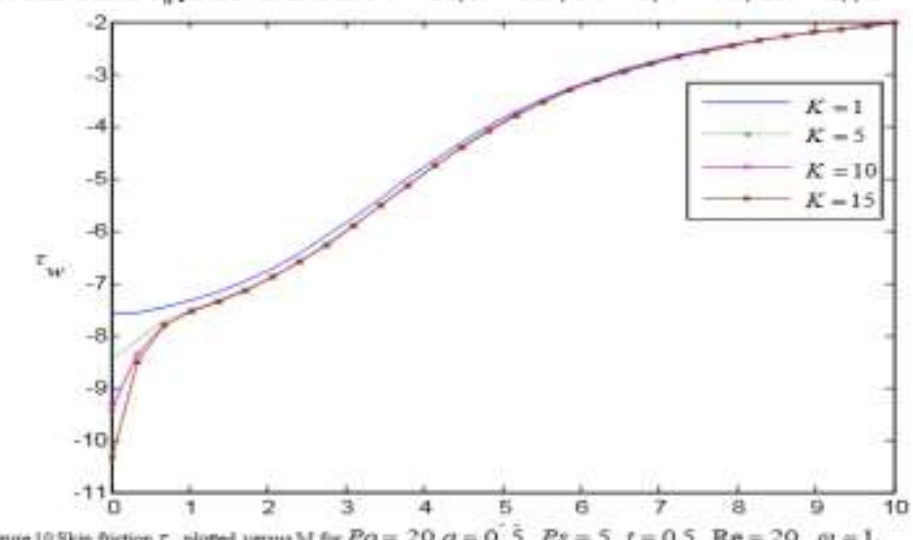

